\title{
A novel mutation in the vraS gene of Staphylococcus aureus contributes to reduce susceptibility against daptomycin
}

\author{
Jie Su${ }^{1}$, Maki Iehara, Jyunichiro Yasukawa, Yasuhiko Matsumoto, Hiroshi Hamamoto and Kazuhisa Sekimizu
}

The Journal of Antibiotics (2015) 68, 646-648; doi:10.1038/ja.2015.42; published online 22 April 2015

Staphylococcus aureus is a Gram-positive bacterium that infects the skin and soft tissue in humans. ${ }^{1,2}$ The spread of drug-resistant pathogens, such as methicillin-resistant $S$. aureus strains (MRSA), is a major clinical problem resulting in treatment failure. ${ }^{3-5}$ The development of novel antibiotics to effectively treat resistant strains is urgently desired. Daptomycin, a cyclic lipopeptide antibiotic, was recently approved for the treatment of MRSA infection. ${ }^{6}$ The bactericidal action of daptomycin results from disruption of the bacterial membrane integrity depending on the presence of calcium. ${ }^{6}$ Clinical observation of $S$. aureus with reduced susceptibility to daptomycin, however, was recently reported. ${ }^{7}$ To better understand the clinical limitations of daptomycin, it is important to identify the gene mutations involved in daptomycin resistance. For this purpose, we screened strains of $S$. aureus with reduced susceptibility to daptomycin and identified a mutation that contributes to daptomycin susceptibility. Identification by the usual genetic methods, however, was not possible due to the low level reduction of daptomycin susceptibility, thus we attempted to isolate mutants exhibiting both reduced daptomycin sensitivity and temperature sensitivity. ${ }^{8}$ Here we demonstrated that a single mutation (E276K) in the $\mathrm{rraS}$ gene was responsible for both phenotypes.

To obtain mutants of $S$. aureus with reduced susceptibility to daptomycin, we prepared a full growth culture of a wild-type $S$. aureus, RN4220, in Luria Bertani medium (tryptone $10 \mathrm{gl}^{-1}$, yeast extract $5 \mathrm{gl}^{-1}$ and $\mathrm{NaCl} 10 \mathrm{gl}^{-1}$ ) containing $0.2 \%$ ethylmethanesulfonate at $30^{\circ} \mathrm{C}$. The culture was diluted 100 -fold with tryptic soy broth (TSB) and further incubated at $30^{\circ} \mathrm{C}$ for $9 \mathrm{~h}$. The resulting outgrowth was then spread onto TSB agar plates containing daptomycin $(6.4 \mu \mathrm{g}$ $\mathrm{ml}^{-1}$ ) and incubated at $30^{\circ} \mathrm{C}$ for 2 days. Among 60 resistant colonies, two strains (D19 and D52) showed a temperature-sensitive phenotype that grew at $30^{\circ} \mathrm{C}$, but not at $43^{\circ} \mathrm{C}$. These mutants exhibited a twofold increase in the daptomycin MIC compared with the parent strain RN4220. To identify the mutated gene responsible for the temperature-sensitive phenotype, we screened a genomic library using a previously described method. ${ }^{8}$ Transformants that grew at $43{ }^{\circ} \mathrm{C}$ were isolated, followed by plasmid extraction and sequencing of the inserted genome fragment. The plasmids complemented both phenotypes: temperature sensitivity and reduced daptomycin susceptibility. We found that a region containing the vraS gene was responsible for the complementation. We determined the mutated position in the $v r a S$ gene of the mutant genome by sequencing (primers; 5'-GCGACCTACATATTGACT-3', 5'-GCCTTCACCAACTACTTC-3' and $5^{\prime}$-ACCACCATTAGACCAACA- $3^{\prime}$ ) and revealed that both mutants had the same mutation (g826a) in the vraS gene, which results in a single amino acid substitution (E276K).

To further confirm that this mutation in the vraS gene accounted for both reduced daptomycin susceptibility and temperature sensitivity, we performed a phage transduction analysis as described previously. ${ }^{9}$ As a donor strain for phage transduction, we used a strain harboring an erythromycin-resistant marker inserted in the SA1705 gene (M1705), ${ }^{10}$ located $2.7 \mathrm{~kb}$ from the $v \mathrm{raS}$ gene. For the first phage transduction, the wild-type vraS gene was introduced into the mutant D52 (Dap $\left.{ }^{r}, T S\right)$. Phages from the donor were used to infect the mutant D52, and transductants were selected based on the erythromycin-resistant phenotype. Among 20 transductants, 8 strains restored daptomycin sensitivity and temperature resistance, whereas the remaining transductants exhibited both reduced daptomycin susceptibility and temperature-sensitivity phenotypes. We next used S. aureus RN4220 with the wild-type vraS gene as the recipient strain in the second phage transduction experiment. A transductant from the first phage transduction with reduced daptomycin susceptibility and temperature-sensitive phenotypes was used as a donor strain. The findings indicated that the temperature-sensitive and reduced daptomycin-sensitive phenotypes were associated with the mutation in the vraS gene (Supplementary Table 1). On the basis of these results, we concluded that a single mutation in the $\mathrm{rraS}$ gene (g826a) was responsible for both the temperature-sensitive and reduced daptomycin-sensitive phenotypes. We used transductants isolated by the second phage transduction experiment for further analysis. 
Table 1 MIC of antimicrobial agents against second phage transductants

\begin{tabular}{lccccccc}
\hline \multirow{2}{*}{ Genotype of vraS } & \multicolumn{9}{c}{ MIC $\left.(\mu \mathrm{g} \mathrm{m})^{-1}\right)$} \\
\cline { 2 - 7 } & Daptomycin & Vancomycin & Chloramphenicol & Linezolid & Ciprofloxacin & Tetracycline & Lysocin E \\
\hline Wild type & 0.25 & 0.8 & 4 & 1.6 & 0.4 & 0.1 \\
Mutant (E276K) & 1 & 1.6 & 4 & 1.6 & 0.4 & 0.1 \\
\hline
\end{tabular}

These MICs were determined by Clinical and Laboratory Standards Institute standard method using CAMHB (cation-adjusted Mueller-Hinton broth; Ca ${ }^{++} 50$ mg $\left.I^{-1}\right)^{16}$ and using two different transductants for each genotype, and the same values were obtained.

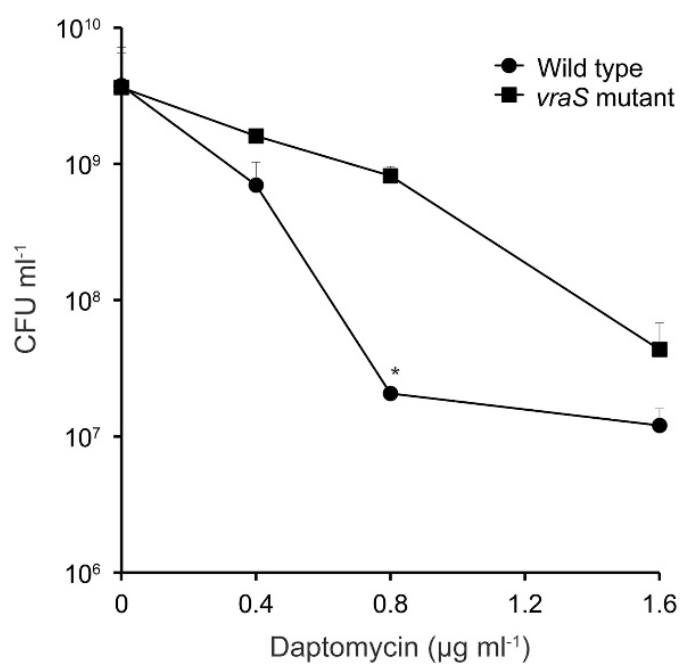

Figure 1 Bactericidal effect of daptomycin against the Staphylococcus aureus mutant. S. aureus was incubated in cation-adjusted Muellar-Hinton Broth (CAMHB: $\mathrm{Ca}^{++} 50 \mathrm{mgl}^{-1}$ ) containing daptomycin (0, 0.4, 0.8 and $1.6 \mu \mathrm{g} \mathrm{ml}^{-1}$ ) at $30^{\circ} \mathrm{C}$ for $1 \mathrm{~h}$, and then the culture aliquots were collected and plated on LB10 plates according to standard method. ${ }^{15}$ Cell viability was determined by counting the colony forming units per milliliter. Circle: wild-type vraS strain of the second phage transductant, square: vraS mutant of the second phage transductant. Statistically significant differences were analyzed by Student's one-tailed $t$-test $\left({ }^{*} P<0.01\right)$ from three independent experiments. The error bars are standard deviation.

The MIC of daptomycin against the vraS mutants was fourfold higher than that against the wild-type vraS strain (Table 1).

We next compared the bactericidal effects of daptomycin on the wild-type $\mathrm{raS}$ strain and the $\mathrm{raS}$ mutant strain. The number of surviving cells of the wild-type vraS strain was significantly reduced by two orders of magnitude after exposure to daptomycin at $0.8 \mu \mathrm{g} \mathrm{ml}^{-1}$, whereas reduction of the $\mathrm{vraS}$ mutation (g826a) was less than an order of magnitude (Figure 1). This finding suggests that this single mutation in the vraS gene induced resistance against the bactericidal activity of daptomycin. We further examined the sensitivity of the vraS gene mutant to other antibiotics. The MIC value of vancomycin against the vraS gene mutants was twofold higher, whereas the sensitivity of the vraS gene mutants to chloramphenicol, linezolid, ciprofloxacin and tetracycline was indistinguishable (Table 1). These results suggest that the single point mutation of the vraS gene in $S$. aureus reduced the susceptibility to both daptomycin and vancomycin. To our knowledge, this is the first report that a single mutation (g826a) in the vraS gene is involved in reduction of the daptomycin and vancomycin susceptibility, and temperature sensitivity.

VraS functions as a membrane sensor in a two-component system that responds to cell wall stress induced by antibiotics such as vancomycin. ${ }^{11}$ Previous studies reported that disruption of the vraS gene in S. aureus increased susceptibility to daptomycin and overexpression of the $v \mathrm{raS}$ gene restored resistance to daptomycin. ${ }^{12,13}$ In addition, two amino acid substitutions in the vraS gene were recently reported to cause both vancomycin and daptomycin resistance. ${ }^{14}$ In this study, we demonstrated that a novel single mutation in the vraS gene contributed to reduction of the daptomycin and vancomycin susceptibility of $S$. aureus. These findings imply that $S$. aureus with reduced daptomycin susceptibility has decreased susceptibility to vancomycin. In addition, the vraS gene mutant did not change susceptibility to lysocin E, which has membrane-damaging effects in S. aureus by a different mechanism from daptomycin (Table 1). ${ }^{8}$ This finding suggests that lysocin $\mathrm{E}$ does not show cross-resistance with antibiotics such as vancomycin and daptomycin.

In conclusion, a single novel mutation (g826a) in the vraS gene is involved in a mechanism of reduced daptomycin susceptibility. We demonstrated that this mutation causes reduced vancomycin sensitivity. This single mutation (g826a) in the vraS gene may cause excess activation of the VraRS signaling pathway, although the precise mechanism requires further investigation.

\section{CONFLICT OF INTEREST}

KS is a consultant for Genome Pharmaceutical Institute. The remaining authors declare no conflict of interest.

\section{ACKNOWLEDGEMENTS}

This study was supported by Genome Pharmaceuticals Institute, Grant-in-Aid for Scientific Research on Innovative Areas 'Chemical Biology of Natural Products' (24102510) for KS and (26102714) for HH from MEXT, and in partly by Grant-in-Aid for Young Scientists (A) (24689008) for HH from JSPS. We thank Ms Kiyomi Kyogoku (University of Tokyo), Dr Keiko Kataoka (University of Tokyo) and Mr Yoshihiro Nakatani (Genome Pharmaceuticals Insititute) for technical assistance.

1 Garcia-Lara, J, Masalha, M \& Foster, SJ. Staphylococcus aureus: the search for novel targets. Drug Discov. Today 10, 643-651 (2005).

2 Lowy, FD. Staphylococcus aureus infections. N. Engl. J. Med. 339, 520-532 (1998).

3 Chambers, HF \& Deleo, FR. Waves of resistance: Staphylococcus aureus in the antibiotic era. Nat. Rev. Microbiol. 7, 629-641 (2009).

4 Demain, AL \& Sanchez, S. Microbial drug discovery: 80 years of progress. J. Antibiot. (Tokyo) 62, 5-16 (2009).

5 Kaito, C \& Sekimizu, K. A silkworm model of pathogenic bacterial infection. Drug Discov. Ther. 1, 89-93 (2007).

6 Bayer, AS, Schneider, T \& Sahl, HG. Mechanisms of daptomycin resistance in Staphylococcus aureus: role of the cell membrane and cell wall. Ann. N. Y. Acad. Sci. 1277, 139-158 (2013).

7 Baltz, RH. Daptomycin: mechanisms of action and resistance, and biosynthetic engineering. Curr. Opin. Chem. Biol. 13, 144-151 (2009).

8 Hamamoto, $\mathrm{H}$ et al. Lysocin $\mathrm{E}$ is a new antibiotic that targets menaquinone in the bacterial membrane. Nat. Chem. Biol. 11, 127-133 (2015)

9 Novick, RP. Genetic systems in staphylococci. Methods Enzymol. 204, 587-636 (1991).

10 Kaito, $\mathrm{C}$ et al. Silkworm pathogenic bacteria infection model for identification of novel virulence genes. Mol. Microbiol. 56, 934-944 (2005) 
11 Boyle-Vavra, S, Yin, S, Jo, DS, Montgomery, CP \& Daum, RS. VraT/YvqF is required for methicillin resistance and activation of the VraSR regulon in Staphylococcus aureus. Antimicrob. Agents Chemother. 57, 83-95 (2013).

12 Mehta, S et al. VraSR two-component regulatory system contributes to mprF-mediated decreased susceptibility to daptomycin in in vivo-selected clinical strains of methicillin-resistant Staphylococcus aureus. Antimicrob. Agents Chemother. 56, 92-102 (2012).

13 Muthaiyan, A, Silverman, JA, Jayaswal, RK \& Wilkinson, BJ. Transcriptional profiling reveals that daptomycin induces the Staphylococcus aureus cell wall stress stimulon and genes responsive to membrane depolarization. Antimicrob. Agents Chemother. 52, 980-990 (2008)
14 Berscheid, A et al. Generation of a vancomycin-intermediate Staphylococcus aureus (VISA) strain by two amino acid exchanges in VraS. J. Antimicrob. Chemother. 69, 3190-3198 (2014).

15 National Committee for Clinical Laboratory Standards Methods for determining bactericidal activity of antimicrobial agents; approved guideline NCCLS document M26-A, (National Committee for Clinical Laboratory Standards, Wayne, PA, USA, Vol 19, 1999)

16 Clinical and Laboratory Standards Institute Methods for dilution antimicrobial susceptibility tests for bacteria that grow aerobically; approved standard - Eighth edition CLSI document MO7-A8, (Clinical and Laboratory Standards Institute, Wayne, PA, USA, Vol 29, 2009)

Supplementary Information accompanies the paper on The Journal of Antibiotics website (http://www.nature.com/ja) 